\title{
Protección contra la corrosión de aleaciones de aluminio mediante recubrimientos sol-gel
}

\author{
A. CONDE*, A. DURÁN**, J. DE DAMBORENEA* \\ *Centro Nacional de Investigaciones Metalúrgicas CENIM/CSIC. Avenida Gregorio del Amo 8, 28040-Madrid \\ **Instituto de Cerámica y vidrio (CSIC), Carretera de Arganda, Km 24.300 28500 Arganda del Rey (Madrid)
}

En el presente trabajo se estudia la protección frente a la corrosión que proporcionan tres nuevos recubrimientos nanoméricos de baja temperatura de curado obtenidos a partir de silanos modificados. La síntesis de estos recubrimientos se realiza a partir de una mezcla de dos soles, GPTS (glicidoxipropiltrimetoxisilano) prehidrolizado y un segundo sol TEOS/MTES. Añadiendo a este sol inicial una disolución coloidal de partículas se obtienen recubrimientos nanoméricos de mayor espesor. En el presente trabajo los recubrimientos particulados se obtuvieron incorporando un $25 \%$ en peso de partículas comerciales de $\mathrm{SiO}_{2}$, Aerosil 300 y Aerosil R972 respectivamente. Una vez sintetizados, se depositaron mediante el método de centrifugado, sobre dos aleaciones de aluminio, 5555 y 3005 empleadas como substratos. El espesor final de los recubrimientos es de orden de 10 ìm, para el recubrimiento sin partículas y algo superiores, 12-14 ìm aproximadamente, para los recubrimientos con partículas. Los resultados electroquímicos indicaron que, en todos los casos, las muestras recubiertas presentaban una mejora en el comportamiento frente a la corrosión respecto de los substratos sin recubrir. La velocidad de corrosión disminuye 3 órdenes de magnitud para los recubrimientos con partículas y ésta disminución es algo inferior para aquellos que no contienen partículas.

Palabras clave: Recubrimientos sol-gel, corrosión, aleaciones de aluminio

\section{Corrosion protection of aluminium alloys by sol-gel coatings}

Present paper studies the corrosion protection of three new nanomeric coatings obtained from modified silanes and cured at low temperatures. The synthesis of these coatings is carried out starting from a mixture of two sols separately prepared: a prehydrolized GPTS (glicidoxipropiltrimetoxisilano) and a second sol TEOS/MTES. Adding to this initial sol a colloidal solution of $25 \%$ in weight of commercial particles of $\mathrm{SiO}_{2}$, Aerosil 300 and Aerosil R972 respectively can be obtained thicker nanomeric coatings. Once synthesized, they were deposited by means of the spin coating method onto two aluminum alloys, 5555 and 3005 used as substrates. The final thickness of the coatings is about $10 \mu \mathrm{m}$, for the coating without particles and slightly higher, 12-14 $\mathrm{m}$ approximately, for the coatings with particles. The electrochemical results indicated that, in all cases, the coated samples presented an improvement in the corrosion behavior in comparison with bare alloys in aggressive media of $\mathrm{NaCl}$. The corrosion rate decreased three orders of magnitude for the coatings with particles and lower for those that do not contain particles.

Keywords: Sol-gel coatings, corrosion, aluminium alloys

\section{INTRODUCCIÓN}

Los recubrimientos sol-gel se presentan como una alternativa para la protección frente a la corrosión de diferentes metales y aleaciones en una amplia variedad de ambientes agresivos $(1,2)$. La versatilidad que ofrece la ruta sol-gel para la obtención de recubrimientos con propiedades y composiciones específicas hace que su ámbito de aplicación sea cada vez mayor.

Desde el punto de vista de la protección frente a la corrosión de los metales y aleaciones, la utilización de la ruta solgel estaba limitada, hasta hace poco, por las altas temperaturas de curado necesarias para sellar el recubrimiento. Temperatura que podían inducir a la sensibilización del substrato y/o a la pérdida de su integridad (3). Sin embargo, la aparición de nuevos métodos de obtención de recubrimientos nanoparticulados a partir de compuestos orgánico-inorgánicos reduce notablemente la temperatura de curado, lo que permite preservar las propiedades mecánicas del substrato metálico. Por otra parte, se disminuye el riesgo de aparición de grietas o defectos originados tanto por las tensiones aparecidas durante el enfriamiento como por los cambios superficiales originados durante el tratamiento de sinterización. Por último, el carácter híbrido de estos recubrimientos permite influir sobre otro tipo de propiedades como las ópticas (transparencia, colorabilidad, resistencia a las manchas), mecánicas (resistencia al desgaste) así como aprovechar la naturaleza más ecológica de los mismos dada la ausencia de disolventes orgánicos durante el proceso.

Estas propiedades hace que los recubrimientos curados a baja temperatura sean un método de protección muy interesante para las aleaciones de aluminio, muy susceptibles a los tratamientos térmicos (4). A pesar de este interés son pocos, todavía, los trabajos que han estudiado el comportamiento frente a la corrosión de las aleaciones de aluminio recubiertas mediante esta tecnología.

En el presente trabajo se caracteriza la naturaleza protectora de tres nuevos recubrimientos nanoméricos, curados a 
$130^{\circ} \mathrm{C}$ durante una hora, y que fueron obtenidos vía sol-gel a partir de un compuesto epoxídico basado en silanos modificados. Los recubrimientos se aplicaron sobre dos aleaciones de aluminio, denominadas AA3005 y AA5555 cuyas principales campos de aplicación se localizan en la industria de la construcción y del automóvil.

\section{DESCRIPCIÓN EXPERIMENTAL.}

Los substratos utilizados eran dos aleaciones base aluminio cuya composición porcentual se recoge en la tabla I.

Previa a la deposición de los recubrimientos los substratos de aluminio se limpiaron y desengrasaron en un baño de ultrasonidos a una temperatura de $65^{\circ} \mathrm{C}$ durante 5 minutos.

Los recubrimientos se obtuvieron a partir de un sol inicial sin partículas y el mismo sol modificado mediante la adición de dos tipos de partículas de sílice coloidal. El sol inicial se preparó en dos etapas, a partir de un silano modificado (3-glicidoxipropiltrimetoxisilano, GPTS) prehidrolizado en medio ácido, al cual se agregó un sol obtenido a partir de MTEOS y TEOS hidrolizado en las mismas condiciones junto a un compuesto orgánico que inicia la polimerización.

A partir de este sol se prepararon otros dos adicionando un 25\% en peso de partículas Aerosil 300 y de Aerosil R972 respectivamente.

Los recubrimientos se obtuvieron por centrifugado y se curaron a $130^{\circ} \mathrm{C}$ durante 1 hora. Acorde con la tabla II, las capas se denominan en adelante A, B y C.

Los espesores obtenidos son de aproximadamente $10 \mu \mathrm{m}$ para el sol A (sin partículas) y de 12-14 um para los soles B y C.

Como referencia, se han realizado ensayos sobre las mismas aleaciones de aluminio recubiertas con el sistema PVDF, empleado principalmente en el pintado de chapa de acero. Este tipo de recubrimientos orgánicos constan de varias capas, lo que proporciona al material base una extraordinaria resistencia frente a la corrosión.

La resistencia a la corrosión de este tipo de recubrimientos se caracterizó en soluciones de $\mathrm{NaCl}$ 0,6M mediante técnicas electroquímicas de corriente continua realizadas con un potenciostato CMS 300 de Gamry Instruments, utilizando la configuración típica de tres electrodos. El electrodo de trabajo estaba constituido por $1 \mathrm{~cm}^{2}$ de la probeta objeto de ensayo y se emplearon un contraelectrodo de platino y un electrodo de referencia de calomelanos saturado (ECS).

Se llevaron a cabo dos tipos de medidas electroquímicas. Por un lado, curvas de polarización en un amplio barrido de potenciales, desde $-1 \mathrm{~V} / \mathrm{ECS}$ a 1,5V/ECS, a una velocidad de barrido de $0.2 \mathrm{mVs}^{-1}$ y por otro, ensayos de polarización potenciostática. Estos se realizaron aplicando pulsos de potencial de 1, 1,25, 1,5, 1,75 y 2 V/ECS de 20 segundos de duración con el fin de determinar el potencial de picadura de los recubrimientos.

\section{RESULTADOS Y DISCUSIÓN}

En primer lugar, se observa que los valores del potencial de corrosión ( $\left.\mathrm{E}_{\text {corr }}\right)$ para las muestras recubiertas se desplazan, en general, hacia valores más nobles. En la tabla III, se recogen los valores de dichos potenciales para las distintas condiciones ensayadas. Como puede observarse, en los recubrimientos con partículas, este desplazamiento es algo mayor aunque no sea especialmente significativo. $\mathrm{El}_{\text {corr }}$ no es una
TABLA I. COMPOSICIÓN (\% EN MASA) DE LAS ALEACIONES DE ALUMINIO EMPLEADAS.

\begin{tabular}{|l|l|l|l|l|l|l|l|l|}
\hline Aleación & Mn & $\mathrm{Mg}$ & $\mathrm{Si}$ & $\mathrm{Fe}$ & $\mathrm{Cu}$ & $\mathrm{Zn}$ & $\mathrm{Cr}, \mathrm{Ti}$ & Al \\
\hline 3005 & 1.2 & $\mathbf{0 . 4}$ & $\mathbf{0 . 6}$ & $\mathbf{0 . 7}$ & $\mathbf{0 . 3 0}$ & $\mathbf{0 . 2 5}$ & $<\mathbf{0 . 1}$ & Resto \\
\hline $\mathbf{5 5 5 5}$ & 1.17 & $\mathbf{0 . 9 6}$ & $\mathbf{0 . 2 1}$ & $\mathbf{0 . 5 2}$ & $\mathbf{0 . 0 5}$ & $\mathbf{0 . 0 3}$ & $<\mathbf{0 . 0 1}$ & Resto \\
\hline
\end{tabular}

TABLA II. RECUBRIMIENTOS ENSAYADOS.

\begin{tabular}{|l|l|}
\hline Recubrimiento & Composición \\
\hline A & GPTS con MTEOS+TEOS \\
\hline B & A $+25 \%$ Aerosil 300 \\
\hline C & A $+25 \%$ Aerosil R972 \\
\hline
\end{tabular}

TABLA III. POTENCIALES DE CORROSIÓN DE LAS MUESTRAS ENSAYADAS.

\begin{tabular}{|l|l|l|}
\hline & Al 3005 & Al5555 \\
\hline & $\mathbf{E}_{\text {COR }}$ & E $_{\text {COR }}$ \\
\hline Sin recubrimiento & -881 & -833 \\
\hline A & -769 & -911 \\
\hline B & -669 & -688 \\
\hline C & -752 & -712 \\
\hline
\end{tabular}

magnitud que permita caracterizar el fenómeno de corrosión en un sistema dado (5) y su variación está determinada por distintos factores como son la naturaleza del metal, el medio o las reacciones electródicas que tuvieran lugar. En los recubrimientos sol-gel estos valores vendrán, además, afectados por las propiedades de la capa y por la densidad de defectos presentes en ella. Resulta evidente que si en el recubrimiento no existieran defectos que pusieran en contacto al metal con el medio, el potencial medido vendría definido por una serie de variaciones aleatorias como las mostradas en otros recubrimientos cerámicos vitrificados (6). Sin embargo, en este caso, como en otros recogidos en la bibliografía, se describe un aumento del potencial de corrosión para distintos materiales base con recubrimientos sol-gel. Así, Thim y col (7), trabajando sobre $\mathrm{Al}(98 \%)$ recubierto de sílice constatan el fenómeno sin explicar la causa. Vasconcelos y col (8) encuentran la misma tendencia en aceros inoxidables con recubrimientos base TEOS, achacándola a la menor concentración de grupos hidroxilo en las películas, por efecto de la temperatura de curado, $200^{\circ} \mathrm{C}$, lo que aumenta la estabilidad química y las propiedades mecánicas del recubrimiento. En nuestro caso, creemos que dichas variaciones sólo parecen indicar cambios en la interfase metal/electrolito. Dado que, como ya se ha comentado, el potencial de corrosión es un parámetro característico de un metal en un medio agresivo, los cambios de dicho parámetro deberían ser indicativos de cambios de composición de la superficie metálica o de cambios locales en la química del sistema. En el sistema aquí estudiado, estas variaciones podrían deberse tanto al proceso de obtención del recubrimiento, que puede dejar alguna especie adsorbida sobre la superficie metálica, o a una alteración local de la química del recubrimiento, pero no a cambios inducidos por el propio recubrimiento sobre la superficie metálica.

En las figuras 1 y 2, se presentan las curvas de polarización correspondientes a los tres recubrimientos ensayados junto con la curva del material sin recubrir y la del sistema de referencia cuyas propiedades quieren ser alcanzadas (aluminio protegido mediante el sistema de pintura PVDF). Estos ensayos se han realizado para cada substrato de aluminio y en 

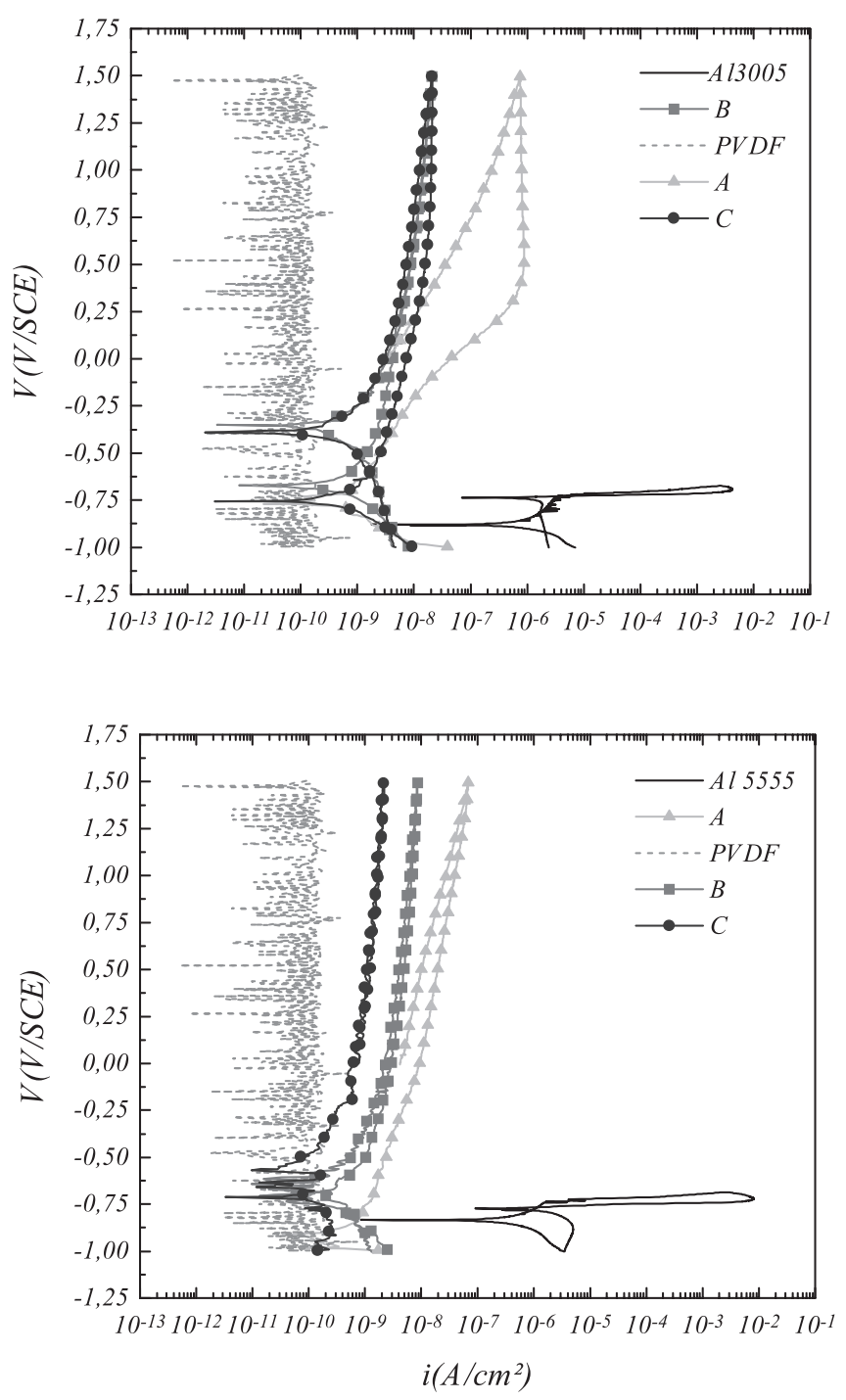

Figura 1: Curvas de polarización de los 3 epóxicos sobre cada substrato. (a) Al5555 y (b) 3005

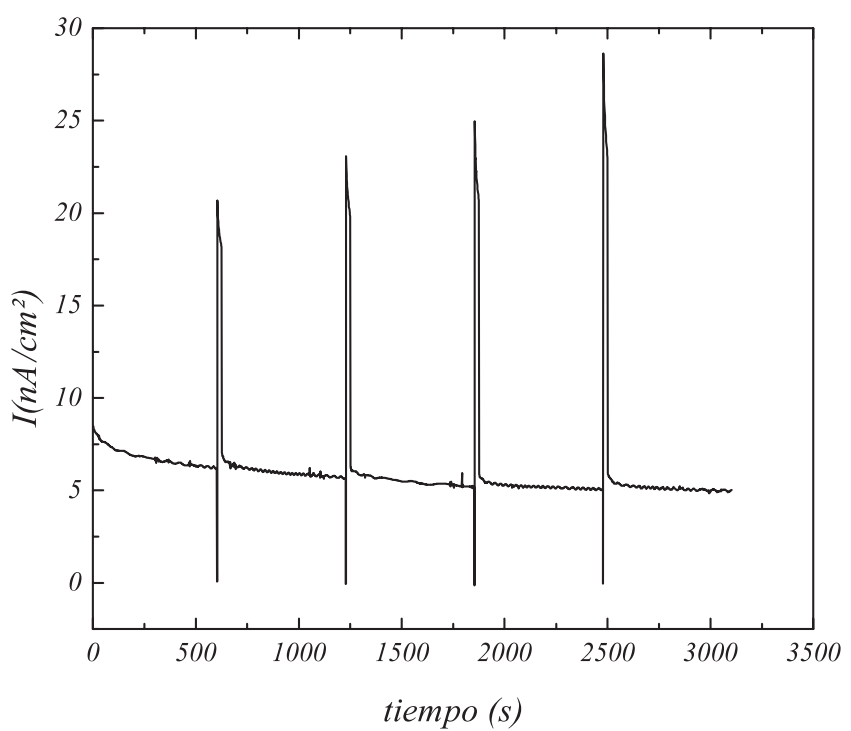

Figura 2: Ensayo de polarización potenciostática solución acuosa de $\mathrm{NaCl}$ y de ácido acético, respectivamente.

Para las dos aleaciones estudiadas los ensayos realizados en $\mathrm{NaCl} 3.56 \%$ en peso, , las probetas recubiertas presentan unas curvas de polarización caracterizadas por un tramo de pasividad que se describe en un amplio intervalo de potenciales, que va desde el $\mathrm{E}_{\text {corr }}$ hasta $1.5 \mathrm{~V} / \mathrm{SCE}$, potencial de retorno elegido no por rotura del recubrimiento sino para evitar reacciones secundarias que enmascaren el proceso. Como puede verse, las densidades de corriente obtenidas para los recubrimientos sol-gel $\left(10^{-9} \mathrm{~A} \mathrm{~cm}^{-2}\right)$ son casi tres órdenes de magnitud menores que las correspondientes al aluminio desnudo $\left(10^{-6} \mathrm{~A} \mathrm{~cm}^{-2}\right)$. Estos valores son, sin embargo, superiores a los encontrados en las probetas recubiertas con el sistema PVDF $\left(<10^{-10} \mathrm{Acm}^{-2}\right)$. Este último valor no debe tomarse como real dado que está en el límite de sensibilidad del aparato de medida y lo único que realmente indica esta medida es que el material se está comportando como un dieléctrico.

Las densidades de corriente de los recubrimientos B y C son menores que las de recubrimiento A. Ello se debe a que la adición de partículas permite obtener recubrimientos más densos y de mayor espesor, ya que la presencia de las partículas disminuye el efecto de la contracción del recubrimiento (durante su enfriamiento desde la temperatura de curado), lo que favorece la menor aparición de grietas.

Esta tendencia queda reflejada más claramente en los ensayos de polarización potenciostática en donde se observa que únicamente en los casos en los que existe un defecto o grieta en el recubrimiento se inicia el ataque del material de la base. En general, los materiales protegidos mediante recubrimientos con partículas no presentan ataque por picaduras cuando se les aplica pulsos de 1, 1,25, 1,5, 1,75, 3 V/SCE de 20 segundos de duración. Como se aprecia en la figura 2, tras la aplicación de estos escalones de potencial los valores de la intensidad de corriente, descienden hasta recuperar los valores iniciales de corriente, del orden de los $\mathrm{nA} / \mathrm{cm}^{2}$. Sin embargo, en aquellos casos en los que un defecto esta presente en el recubrimiento (figura 3), la densidad de corriente no recupera el valor inicial tras la aplicación del escalón de potencial, sino que crece de forma continua como consecuencia del proceso de disolución activa del substrato de aluminio.

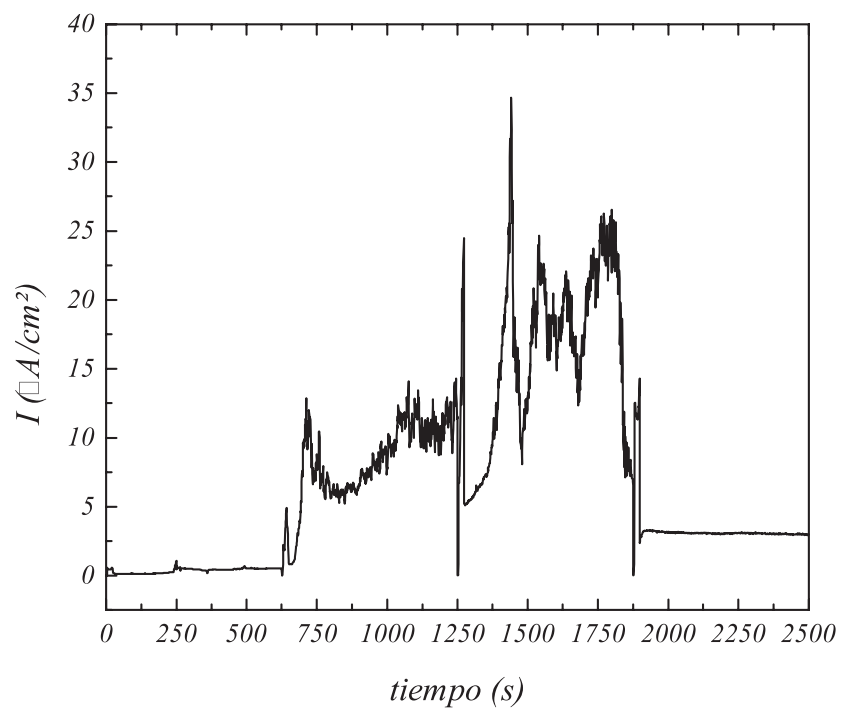

Figura 3: Ensayo de polarización potenciostática.El aumento de la corriente tras la aplicación del pulso de potencial indica la formación de una picadura. 
Las propiedades protectoras de estos recubrimientos se mantienen incluso en aquellos casos en donde la agresividad del medio es mayor. En la figura 4 se recogen las curvas de polarización realizadas en una solución de ácido acético al 10\%. En este medio, y como cabría esperar, las muestras desnudas describen una curva de polarización que revelan un comportamiento muy activo del aluminio, debido a la disolución de la capa óxido protectora en valores de $\mathrm{pH}$ ácidos, lo que se traduce en una elevada velocidad de corrosión.

Las muestras protegidas mediante los recubrimientos nanoméricos, revelan que los recubrimientos particulados, sobre todo el sistema C, proporciona una mayor protección frente a la corrosión, que se traduce en una mayor disminución de la velocidad de corrosión.

En todos los casos, las curvas de polarización muestran la misma tendencia que en la solución de $\mathrm{NaCl} 3.56 \%$; es decir, un comportamiento pasivo de las muestras recubiertas, aunque los tramos de pasividad sean más cortos en este medio. Los valores de la densidad de corriente de pasividad continúan siendo muy bajos $\left(10^{-9} \mathrm{Acm}^{-2}\right)$, lo que pone de manifiesto la baja velocidad de corrosión del material. Sin embargo, la mayor agresividad de la solución induce un ataque corrosivo más extenso en el substrato metálico cuando el electrolito accede a través de los defectos del recubrimiento, provocando, incluso, la delaminación del recubrimiento. Tal como se observó en el examen visual tras la finalización del ensayo, la superficie ensayada presentaba puntos de ataque como los que se recogen en la macrografía de la figura 5. Esta imagen, similar a la obtenida en soluciones de $\mathrm{NaCl}$, constata el desarrollo de un ataque localizado como consecuencia de la existencia de una grieta en el recubrimiento a través de la cual el medio agresivo podía penetrar y atacar al sustrato de aluminio. Por esta razón, aparece tan claramente definido un potencial de ruptura tanto en las probetas protegidas por el sistema A como en el B. El sistema C mantiene prácticamente el mismo comportamiento en las dos soluciones ensayadas lo que parece indicar la existencia de un posible efecto inhibidor del recubrimiento sobre la superficie de las aleaciones de aluminio.

\section{CONCLUSIONES}

De acuerdo con los resultados anteriores, se concluye que los recubrimientos sol-gel proporcionan una elevada resistencia a la corrosión a las aleaciones de aluminio 3005 y 5555 en medios de elevada agresividad. Dentro de estos recubrimientos, los que poseen partículas proporcionan una mayor protección a los substratos en comparación con los que no las tienen. A pesar de que la adición de las mismas podría llevar a pensar en un efecto pernicioso debido a que supone un aumento del carácter inorgánico y por tanto una disminución de su flexibilidad. Sin embargo, los resultados muestran que la incorporación de partículas no contribuye a una mayor tendencia a la aparición de grietas, a través de las cuales el medio agresivo sería capaz de alcanzar al substrato metálico e iniciando el proceso corrosivo. Al contrario, el sistema $\mathrm{C}$ se presenta como una alternativa real para la protección del aluminio incluso en soluciones de $\mathrm{pH}$ ácido, quizá debido a un efecto inhibidor del propio recubrimiento sobre las superficies que pudieran quedar desnudas durante el proceso obtención del mismo.
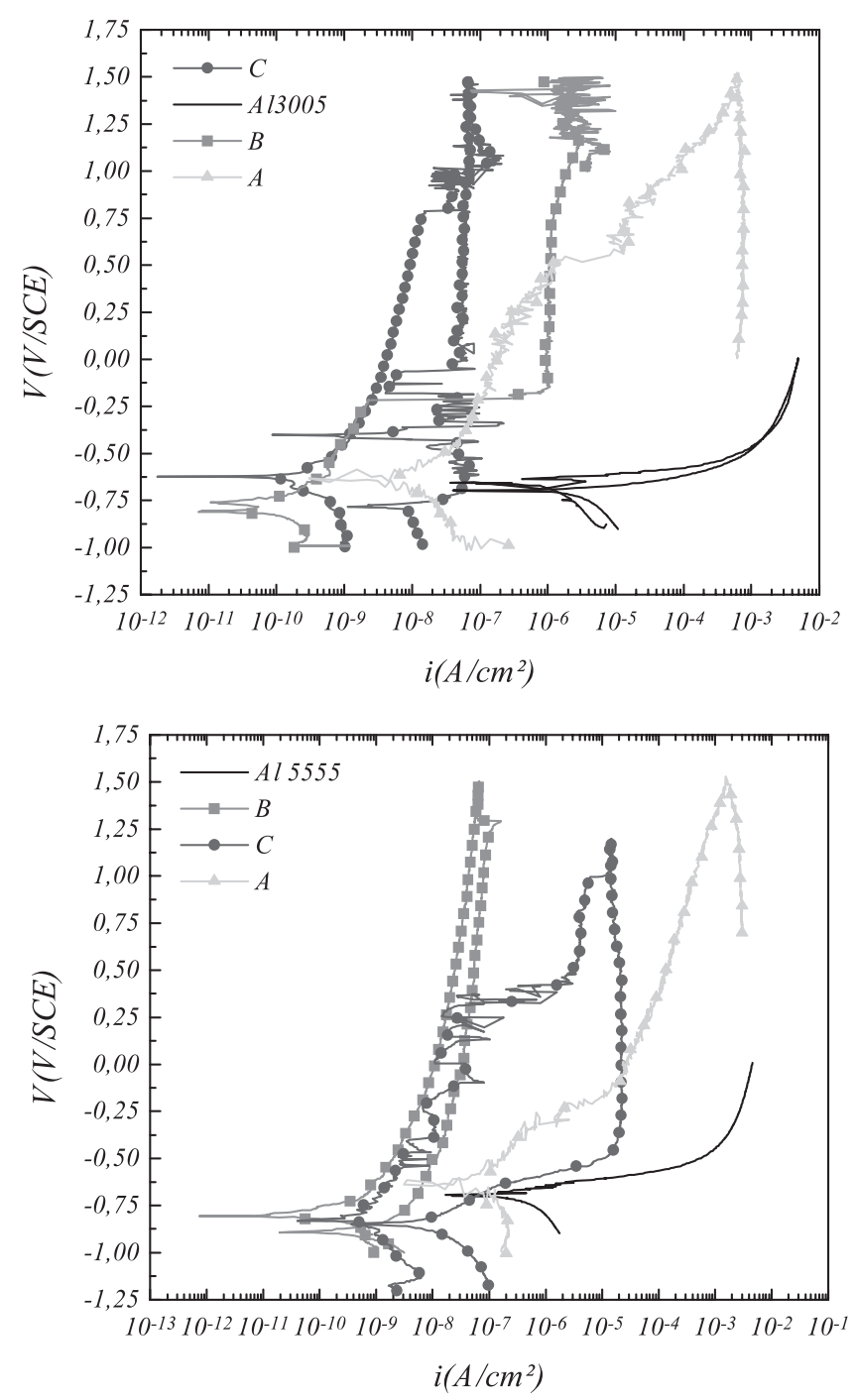

Figura 4: Curvas de polarización en ácido acético 10\%. (a) Al5555 y (b) 3005

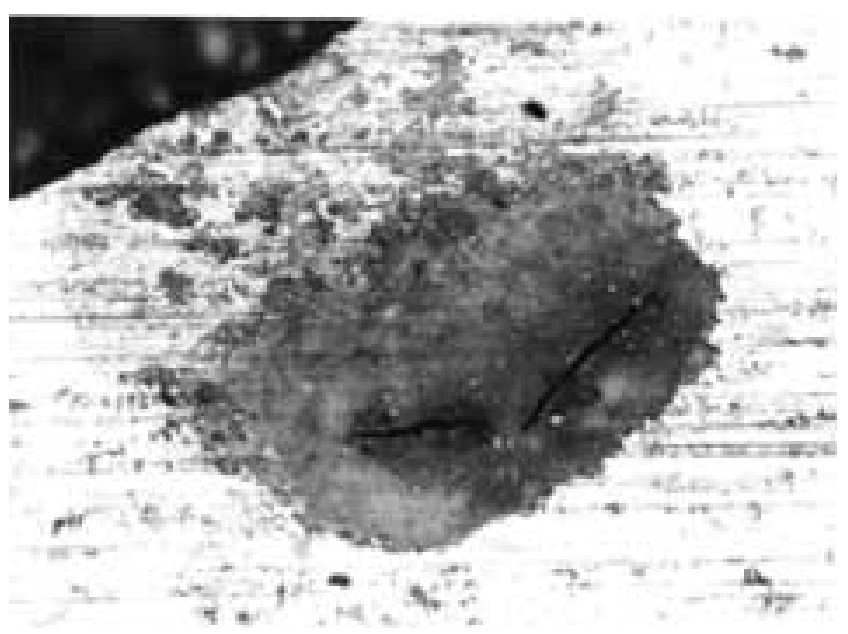

Figura 5: Micrografía óptica de la picadura aparecida tras el ensayo de polarización potenciostática. 


\section{AGRADECIMIENTOS}

Los autores quieren agradecer al proyecto BRITE BE-5111: "High Performance protection with sol-gel coatings on metals and enamels" por financiar parte de este estudio.

\section{BIBLIOGRAFIA}

1. J. Gallardo, P. Galliano, A. Durán, "Bioactive and protective sol-gel coatings on metals for orthopaedic prostheses", J. Sol-Gel Sci. Technol. 21, 65-74 (2001). 2.- M. J. Drews, M. Williams, M. Barr, "The corrosion of sol-gel-coated type 316 SS in chlorinated SC water", Ind. Eng. Chem. Res.39, 4772-4783 (2000).
3.- J. de Damborenea, N. Pellegri, O. Desanctis, A. Durán, "Electrochemicalbehavior of sio2 sol-gel coatings on stainless-steel", J. Sol-Gel Sci. Technol. 4, 239-244(1995).

4.- H. Schmidt, S. Langenfeld, R. Nass, "A new corrosion protection coating system for pressure-cast aluminium automotive parts" Mater. Des. 18, 309-313 (1997).

5.-L. Krivián, "Meaning and measurement of corrosion potential", Br. Corros. J 26, 191-194 (1991)

6.- A. Conde, J. de Damborenea, “ Monitoring of vitreous degradation by electrochemical noise" Surf. Coat. Tech. 150, 212-217 (2002).

7.- G. P. Thim, M. A. S. Oliveira, E. D. A. Oliveira, F. C. L. Melo, "Sol-gel silica film preparation from aqueous solutions for corrosion protection", J. NonCryst. Solids 273, 124-128 (2000).

8.- D. C. L. Vasconcelos, J. A. N. Carvalho, M. Mantel, W. L. Vasconcelos, "Corrosion resistance of stainless steel coated with sol-gel silica J. Non-Cryst. Solids 273, 135-139 (2000)

\section{Materials Transfer Technology Event (MTTE)}

Dentro del marco del VII Congreso Nacional de Materiales, la Dirección General de Investigación de la Comunidad de Madrid, a través del Centro de Enlace para la Innovación (IRC) y el Círculo de Innovación de Materiales y Tecnologías de la Producción (CIMT) organiza una jornada especial de Transferencia de Tecnología en el área de Materiales.

\section{OBJETIVOS}

El principal objetivo de esta jornada es promover la aproximación entre investigadores y responsables empresariales para incrementar el conocimiento mutuo y la posible transferencia de las capacidades científicas y tecnológicas desde los ambientes académicos a los sectores socioeconómicos.

Las principales actividades serán, por una parte, la difusión de las actividades del Círculo de Innovación de Materiales y Tecnologías de la Producción de la Comunidad de Madrid mediante un debate sobre Vigilancia Tecnológica e Inteligencia Económica. Por otra, la organización de una jornada de Transferencia de Tecnología para establecer acuerdos o proyectos de investigación, desarrollo, producción y comercialización entre participantes mediante la celebración de reuniones bilaterales entre empresas, instituciones y grupos de investigación de acuerdo al perfil de cada participante.

\section{PARTICIPANTES}

-Investigadores españoles y del resto de Europa relacionados con el área de Materiales.

-Empresas europeas de Materiales.

-Inversores y empresas de capital riesgo

\section{PROGRAMA}

JUEVES, 17 DE OCtUBRE DE 2002

8:30-9:00. Recepción de inscritos y entrega de documentación

9:00-9:15 Apertura oficial

9:15-9:45. Dr. Francisco Alonso, Gerente del Círculo de Innovación de Materiales y Tecnologías de la Producción (CSIC-Comunidad de Madrid).

9:45-10:00. D. Juan Manuel Pérez Pérez, adjunto al Director del Vivero Virtual de Empresas de la Comunidad de Madrid.

10:00-10:15. D. José de la Sota, Director del Centro de Enlace de Madrid.

10:15-10:30. D. Eduardo Castañeda Díaz, Departamento de Cooperación Internacional (Dirección de Programas Internacionales), CDTI. Financiación para la promoción internacional de transferencia de tecnología.

10:30-11:30. Relaciones exitosas entre OPIs y empresas de tres sectores del área de materiales:

$$
\begin{aligned}
& \text {-Polímeros } \\
& \text {-Cerámica } \\
& \text {-Metalurgia }
\end{aligned}
$$
Café

11:30-12:00

Transferencia de Tecnología. Reuniones bilaterales.

14:00-16:00. Comida

16:00-19:30. Transferencia de Tecnología. Reuniones bilaterales.

INFORMACIÓN DE INTERÉS

Lugar: Palacio de Congresos y Exposiciones de Madrid.

Paseo de la Castellana 99

28046 Madrid.

Metro: Santiago Bernabeu (Línea 10).

Consejo Superior de Investigaciones Científicas

Oficina de Transferencia de Tecnología

Almudena Fernández

Teléfono: (+34) 915616800 (Ext: 3231) / 915855352

Fax: (+34) 915855196.

E-mail: CIMTOOTT@CSIC.ES

Toda la información de este evento estará disponible en

www.madrimasd.org

www.secv.es/madridmateriales2002

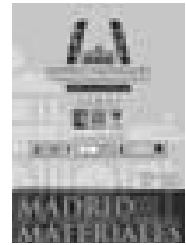

VII Congreso Nacional de Materiales madriథd

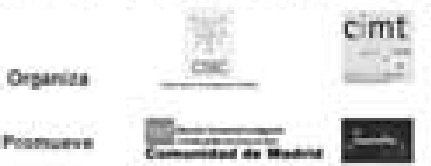

www.madrimasd_org 